\title{
Analysis of Voice Signal Based On High Pitch, Low Pitch, FIIR and IIR Spectrum Suitable For Pervasive Computing Using MatLab
}

\author{
Vijay K Chaudhari, Member IACSIT, Shiv Kumar, Member IACSIT, R. K. Singh and Dinesh Varshney
}

\begin{abstract}
Voice Signal Analysis (VSA) is the process of analyzing the voice signal using the different parameters of DSP. Pitch value define the frequency of the sound, perfect pitch refers to the ability of some persons to recognize the pitch of a voice note without any discernable pitch standard. Formant analysis calculates the peak points of power spectral density for a selected .wav file. In Digital Signal Processing various parameters like Pitch Analysis, Single Echo, Multiple Eco, FIR Reverb, IIR Reverb, Flat Response Reverb, Formant Analysis, Flange, Fade-In, and Fade-Out are mentioned. If the original voice signal is having any of this effect, then it can be visualized by the spectrum of original and effected signal. Here spectrum analysis based on four parameters (High Pitch, Low Pitch, FIIR and IIR) is presented. The proposed analysis is effective for different purposes as to differentiate the original and fake voice, for security purpose etc. and also for pervasive computing for the reason that before this analysis, signal is first compressed to about $50 \%$ with out changing the extension of recorded voice signal.
\end{abstract}

Index Terms-MatLab6.0, DSP Toolbox, .wav warehouse, Algorithms

\section{INTRODUCTION}

Earlier, Chaudhari and coworkers [1] and Shiv Kumar and coworkers [2] develops a method for voice signal compression for limited bits, $\mathrm{N}$ bit signal is used and also the signal is compressed upto $50 \%$ of the original signal with out changing the extension of the compressed signal as illustrated in table-III [2]. We may add that the work of Jeeawoody [3] that is based on limited bits of signal with limited parameters.

The present paper is concerned with the programming methods following MatLab6.0 with DSP Toolbox and mathematical technique Fourier transforms. The Fourier Transform breaks down time signals into frequencies, which can then analyze. We thus analyze the VSA using MatLab

\footnotetext{
Manuscript received December 21, 2009.

Vijay K Chaudhari is Department of Information Technology, Technocrat Institute of Technology-Bhopal (M.P.)-India (Mobile No.: +91-9893181833-e-mail: vijay_ashish@yahoo.com).

Shiv Kumar is with Department of Information Technology, Technocrat Institute of Technology-Bhopal (M.P.)-India (Mobile No.: +91-9827318266, e-mail: shivksahu@rediffmail.com).

Dr. R.K. Singh is Director, MATS University, Raipur (C.G.), India. (Mobile No.: +91-9893623490, e-mail: vcrks@rediffmail.com).

Dr. Dinesh Varshney is with Multimedia Research Department, Multimedia regional centre, Madhya Pradesh Bhoj (Open) University, Khandwa road Campus, Indore (M.P.)-India (Mobile No.: +91-9826076903 email: vdinesh33@rediffmail.com)
}

Toolbox. Spectrum is a function that is used frequently in MatLab to look at the spectral envelope of a recorded sinusoidal wave. Spectrum takes numbers of vectors from a .wav file and the sampling frequency of that file. The function outputs of FFT of the .wav file is plotted in graph format. Background noise was removed up to maximum extent from a signal by the application of a $3^{\text {rd }}$ order Butterworth filter. A code was then developed to compare the pitch spectrum and formant of a known speech file to $\mathrm{N}$ bit unknown speech files and chooses for top 4 matches. The sorting routines performed to sort and compare the average pitch of the reference file with all other files, compare the formant vector of the reference file to all wav files, sort for the top 4 average pitch correlations and then sort these files by formant vectors, and finally sort the top 2 formant vector correlations and then sort these by average pitch.

[Table-III: Result Analysis for Male and Female Category Voice Signal]

\begin{tabular}{|c|c|c|c|c|c|c|c|c|}
\hline \multicolumn{2}{|c|}{ Input File } & \multirow{2}{*}{ 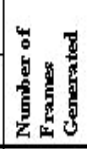 } & \multirow[b]{2}{*}{ Entropy } & \multirow[b]{2}{*}{ SNR } & \multirow[b]{2}{*}{ 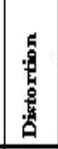 } & \multicolumn{2}{|c|}{ Compressed File } & \multirow{2}{*}{ 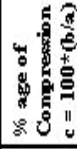 } \\
\hline $\begin{array}{l}\text { Name } \\
\text { (wav) }\end{array}$ & \begin{tabular}{|c|} 
Size \\
(a) \\
in KB \\
\end{tabular} & & & & & $\begin{array}{l}\text { Name } \\
\text { (wav) }\end{array}$ & \begin{tabular}{|c|} 
Size \\
(b) \\
in $\mathrm{KB}$ \\
\end{tabular} & \\
\hline Samplel & 976.00 & 121 & 1835.725 & 3672.466 & 0.453 & Samplel_C & 496 & $50.82 \%$ \\
\hline Sample2 & 1464.32 & 183 & 1119.709 & 5409.053 & 0.299 & Sample2_C & 736 & $50.26 \%$ \\
\hline Sample3 & 330.00 & 42 & 241.2136 & 1043.8105 & 1.328 & Sample3_C & 166 & $50.30 \%$ \\
\hline Sample5 & 1228.8 & 153 & 2492.7317 & 5107.2499 & 0.358 & Samples_C & 624 & $50.78 \%$ \\
\hline Sample6 & 656 & 82 & 417.7178 & 2146.0319 & 0.668 & Sample6_C & 328 & $50.00 \%$ \\
\hline
\end{tabular}

Table is taken from [2]]

The development of the speaker identification system began as early as the 1960s with exploration into voiceprint analysis, where characteristics of an individual's voice were thought to be able to characterize the uniqueness of an individual much like a fingerprint.

In the present investigation, we identify the constitutional and unconstitutional characteristic of .wav file. Speaker record the voice and stores it in the .wav warehouse. Now two .wav file will be same if both file is recorded with same sentences by the same speaker otherwise difference must identify by the pitch value and frequency spectrum. This paper is the part of voice comparison system.

\section{PROBLEM IDENTIFICATION}

Earlier Chaudhari and coworkers [1], proposed an approach for VSA and the analysis of the results were not discussed. The present study is an extension of our earlier work [1] for High Pitch, Low Pitch, FIIR, and IIR is included with flow chart, and DFD. 
We may add that Jeeawoody [3] does not considered VSA for $\mathrm{N}$ bits long parameters for security devices.

To get N-bits long security parameters, different DSP parameters are used [1]:

1. Speech Enhancement

2. Pitch Analysis

3. Single Echo

4. Multiple Eco

5. FIR Reverb

6. IIR Reverb

7. Flat Response Reverb

8. Formant Analysis

9. Flange

10. Fade-In

11. Fade-Out

12. Wavelet Comparison

We note that usually after compression of file, extension also gets changed. If file is compressed with same extension [2], then processing will be very fast and display good result. Also, for security purpose, limited parameters are used and there is no consideration for $\mathrm{N}$ bits parameters [3]. With this motivation we attempt VSA for $\mathrm{N}$ bits parameters. Furthermore, we have in mind to check how the device analyze the constitutional and unconstitutional .wav file if special effects are masked in given input .wav file [4-6]. Thus the present analysis focuses on the above problem using the spectrum of original and the effected voice signal.

\section{A. Speech Enhancement}

The file recorded with slower speech and noise in the background was found from the ordered list of speakers is illustrated in Figure-1. Recorded voice signal is then converted to the frequency domain through the use of a shifted FFT and correctly scaled frequency vector [1].

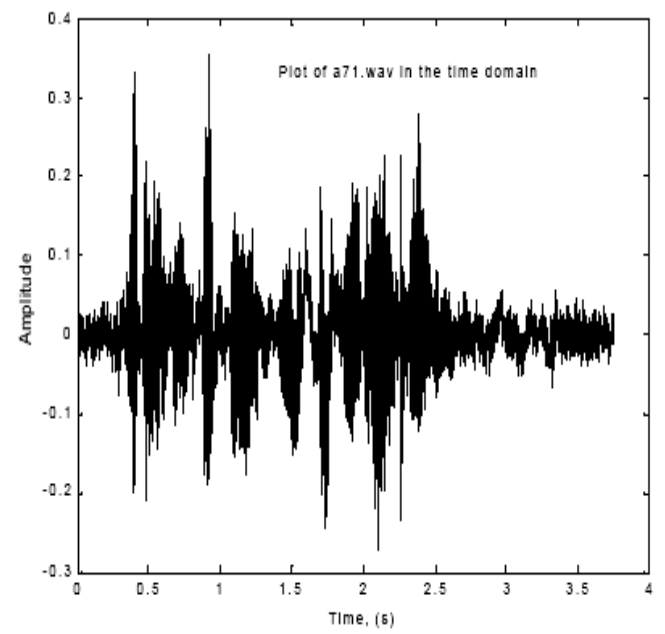

[Fig.-1: Speech Enhancement]

The higher frequency noise components were then removed by application of a 3rd order Butterworth low pass filter with the cutoff chosen to remove as much of the noise signal as possible while still preserving the original signal. Cutoff frequency is that frequency where the magnitude response of the filter is $(1 / 2)^{1 / 2}$. To calculate Butterworth low pass filter output, following equation is used in MatLab (please see
Figure-1).

$$
[b, a]=\operatorname{butter}\left(n, w_{n}\right) \ldots \ldots . .(1)
$$

Equation (2) is then used to design an order $n$ low pass digital Butterworth filter with normalized cutoff frequency Wn. It returns the filter coefficients in length $n+1$ row vectors $b$ and $a$, with coefficients in descending powers of $z$.

$$
H(z)=\frac{B(z)}{A(z)}=\frac{b(1)+b(2) z^{-1}+\ldots+b(n+1) z^{-n}}{1+a(2) z^{-1}+\ldots+a(n+1) z^{-n}}
$$

The Butterworth filter is a reasonable choice to use as it more closely approximates an ideal low pass filter as the order $\mathrm{n}$ is increased. The resulting filtered signal \& the original noisy signal was then scaled and plotted and to get the final comparison.

\section{B. Pitch Analysis}

Pitch is the representation of a sound wave frequency (the number of cycles per second). The file recorded with slower speech was found from the ordered list of speakers. Pitch analysis was conducted and relevant parameters (Low and High Pitch) were extracted. The average pitch of the entire wav file was computed and plotted as shown in figure. The results of pitch analysis can be used in voice recognition, where the differences in average pitch can be used to characterize a voice file. Pitch defines two parameters [1]:

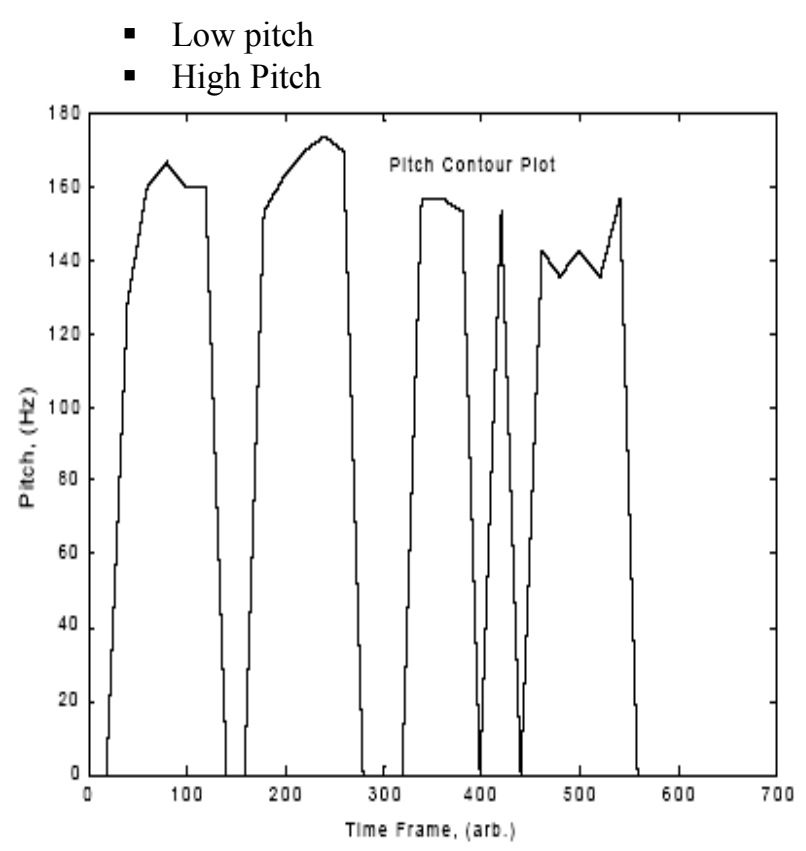

[Fig.-2: Pitch contour plot]

The above modulation standard that the frequency from 440 to $880 \mathrm{~Hz}$, depending on the octave. Low pitch is an effect in which the frequency of wave becomes down to a certain value. High pitch is an effect in which the frequency of wave becomes high to a certain value.

If two .wave file have the frequency $\mathrm{A}=440$ and $\mathrm{B}=547$ $\mathrm{Hz}$ respectively then $\mathrm{A}$ belong to low pitch and $\mathrm{B}$ belong to high pitch standards. 


\section{Single Eco}

The simplest version of delay is an FIR filter through which a signal is played back only one time and is referred as simple delay. Delay time and relative amplitude (and hence relative intensity) for the echoes are two parameters that can be specified [1].

The single echo is nothing but editing a delay in to input signal. An FIR filter can use to edit the delay in input signal. According the need, delay can be increase and decrease for the desired result. This is a MatLab function that makes an approximate calculation of a room's impulse response. The output can then be convolved with an audio clip to produce good and realistic sounding reverb. The effect of delay is achieved when a signal is played and then a modified version of that signal is played back after a period of time, either one time or multiple times, resulting in an echoing effect.

\section{Multiple Eco}

The multiple echoes are nothing but editing a delay in to input signal. A COMB filter can use to edit the delay in input signal. Comb filters are essentially notch filters with deep notches equally spaced in a band of frequencies. The periodic, deep notches make comb filters ideal for applications that need to eliminate specific frequency components. Placing zeros equally spaced around the unit circle at the desired notch locations can design a comb filter. However, the resulting FIR notch filter will exhibit relatively large bandwidth at each notch, which will result in the attenuation of desired frequency components [1].

\section{E. FIIR Reverb}

Reverberation, or reverb, is a slightly more complex FIR form of delay. FIR reverberation is defined as the combined effect of multiple sounds reflections within a room. Reverb is used to digitally simulate a surrounding by modeling echoes that would naturally be present in that environment. The reverberation characteristics of a room are affected by several factors: the shape and size of the room, the materials of which the room is constructed, and the materials present in the room [1].

Filters are electronic circuits that respond to impulses, processing signals by enhancing certain aspects reducing certain aspects. There are several different types of filters. A finite impulse response (FIR) filter is one in which the output signal goes to zero in some finite amount of time. An FIR filter will take as input the current sample in addition to some previous sample An FIR filter has not internal feedback.

\section{F. IIR Reverb}

Reverberation, or reverb, is a slightly more complex IIR form of delay. IIR reverberate involves saving the past values of the output instead of the input values. An infinite impulse response (IIR) filter may run for an arbitrarily long period of time and never have the output go to zero. IIR filter is recursive; i.e., an IIR filter will take its previous output and use it as input for the next output. Reverb is used to digitally simulate a surrounding by modeling echoes that would naturally be present in that environment. An IIR filter creates internal feedback [1].

\section{G. Flat Response Reverb}

Flat-response reverb has a flat response for all values of frequencies. For an all-pass filter the gain is ideally unity for all values. The flat response filter is derived from the all-pass filter for which the poles and the zeros are conjugate reciprocals of each other [1].

\section{H. Formant Analysis}

Formant analysis is performed on slow-recorded voice file. The first five peaks in the power spectral density were returned and the first three can be seen in Fig. 3. The vector position of the peaks in the power spectral density is calculated and can be used to characterize a particular voice file and this technique is used in the waveform comparison section. Power Spectrum is just another mathematical tool for analyzing the signal [1].

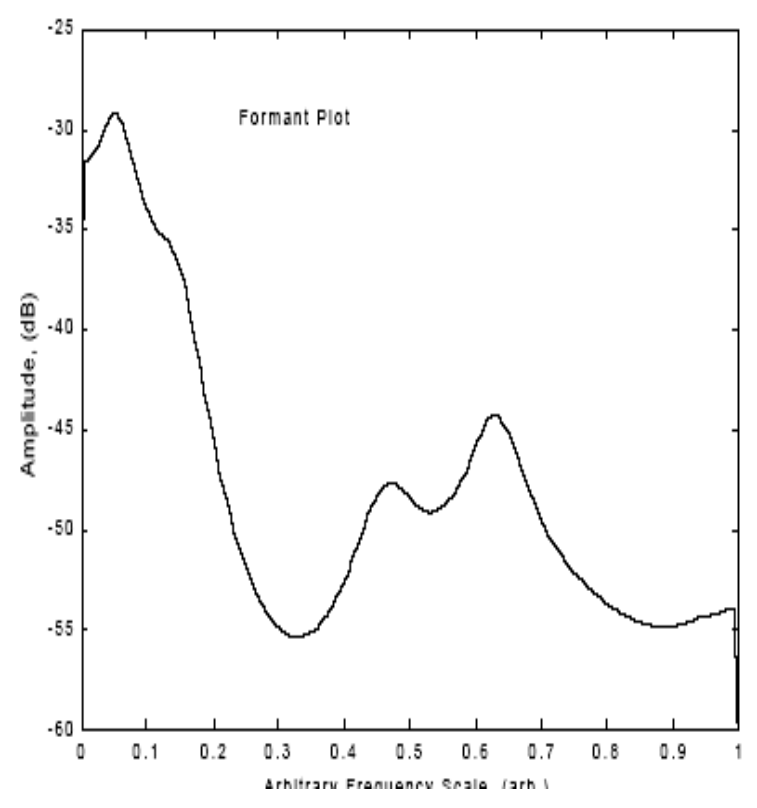

[Fig.-3: Plots of First Few Format]

\section{Flange Analysis}

Flange is used to filter the original signal and try to remove maximum noise from the signal. Noise is the unwanted bits that are edited in between the data. Using FLANGE Effect, one reduces maximum noise from the data [1].

\section{J. Fade In}

The Fade In effect takes care of the soundness of .wav that becomes low to high. One thus analyzes the .wav spectrum from low to high. This effect is used when one the analyze spectrum from low to high [1].

\section{K. Fade Out}

On the other hand, the Fade out effect concerns with the soundness of wav that becomes high to low. One can analyze the .wav spectrum from high to low. The above is used when one desires to analyze spectrum from high to low [1].

\section{Wave Form Comparison}

Following the results and information learned from pitch and formant analysis, a waveform comparison code was developed. Speech waveform files can be thus characterized 
based on various criteria. Average pitch and formant peak position vectors are two such criteria that can be used to characterize a speech file. The slow speech file was used as a reference file. For sorting routines were then written to compare the files. The sorting routines performed to sort and compare the average pitch of the reference file with all other files, compare the formant vector of the reference file to all .wav files, sort for the top 4 average pitch correlations and then sort these files by formant vectors, and finally to sort for the top 2 formant vector correlations and then sort these by average pitch [1].

\section{THE ALGORITHM}

The algorithm for High Pitch analysis, Low Pitch analysis, FIIR analysis, and IIR analysis follows:

\section{A. Algorithm for High Pitch analysis}

Step[1]: Browsed compressed .wav file

Step[2]: Read file size (x) of .wav file $[\mathrm{x}, \mathrm{fs}, \mathrm{bits}]=$ wavread(file)

Step[3]: Read frequency (fs) of .wav file

Step [4]: Compute $\mathrm{N}=$ length $(\mathrm{x})$

Step[5]: Create delay M1 $=0: 0.9: 10$

Step[6]:Compute $\mathrm{x} \_d e c i=x(1: M 1: N)$

Step[7]: Compute $\mathrm{y}=\mathrm{x} \_$deci

Step [8]: Write effected .wav file for value $y$. wavewrite(y,fs, bits, filename)

Step[9]: Plot spectrum for value $\mathrm{x}$ and $\mathrm{y}$.

B. Algorithm for Low Pitch analysis

Step[1]: Browsed compressed .wav file

Step[2]: Read file size (yin) of .wav file [yin,fs, bits]=wavread(file)

Step[3]: Read frequency (fs) of .wav file

Step[4]: Compute $\mathrm{N}=$ length(x)

Step[5]: Create delay M1=0:0.9:10

Step[6]: Compute $\mathrm{x} \_$inter $=\operatorname{zeros}(1, \mathrm{M} 1 * \mathrm{~N})$, and $\mathrm{x}$ inter $(1: \mathrm{M} 1: \mathrm{M} 1 * \mathrm{~N})$

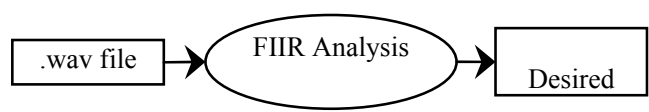

[Fig.-6: 0 Level DFD for FIIR Analysis

Step[7]: $Y=x \_$inter

Step[8]: Write effected .wav file for value $y$.

wavewrite(y,fs, bits, filename)

Step[9]. Plot spectrum for value $\mathrm{x}$ and $\mathrm{y}$.

C. Algorithm for FIIR analysis

Step[1]: Browsed compressed .wav file

Step[2]: Read file size (d) of .wav file $[\mathrm{d}, \mathrm{r}, \mathrm{nbits}]=$ wavread(file)

Step[3]: Read frequency (r) of .wav file

Step[4]: Compute nOfSample $=r * 8$

Step[5]: Compute num $=[0$, zeros(1,noOfSample),1]; den $=[1$, zeros $(1$, noOfSample $),-0.8]$; $\mathrm{d} 1=$ filter(num,den, $\mathrm{d})$;

Step[6]: $\mathrm{Y}=\mathrm{d} 1$
Step[7]: Write effected .wav file for value y. wavewrite(y,fs,bits, filename)

Step[8]. Plot spectrum for value $\mathrm{x}$ and $\mathrm{y}$.

D. Algorithm for IIR analysis

Step[1]: Browsed compressed .wav file

Step[2]: Read file size (d) of .wav file $[\mathrm{d}, \mathrm{r}, \mathrm{nbits}]=$ wavread(file)

Step[3]: Read frequency (r) of .wav file

Step[4]: Compute $\mathrm{R}=$ ceil( $\left(\mathrm{r}^{*} 70 \mathrm{e}-3\right)$;

Step[5]: Compute num $=[0.5, \operatorname{zeros}(1, \mathrm{R}), 1]$; $\operatorname{den}=[1, \operatorname{zeros}(1, \mathrm{R}), 0.5]$; $\mathrm{d} 1=$ filter(num,den, $\mathrm{d}$ );

Step[6]: $Y=d 1$

Step[7]: Write effected wav file for value y. wavewrite(y,fs, bits, filename)

E. O-Level DFD for High Pitch Analysis

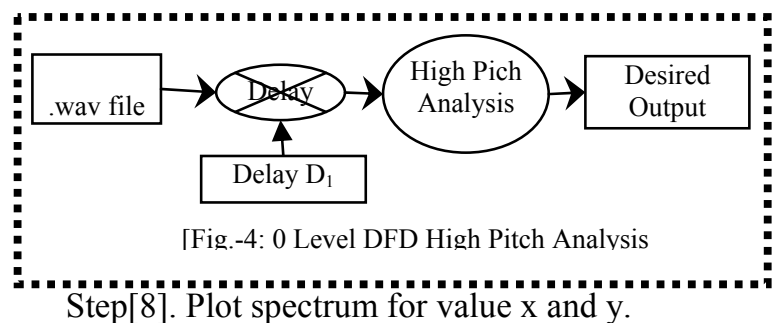

F. O-Level DFD for Low Pitch Analysis

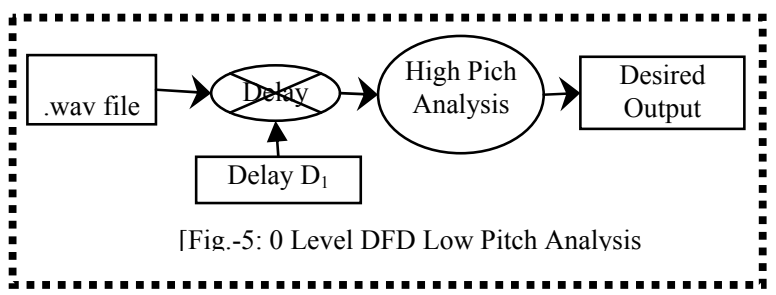

G. 0-Level DFD for FIIR Analysis

H. O-Level DFD for IIR Analysis

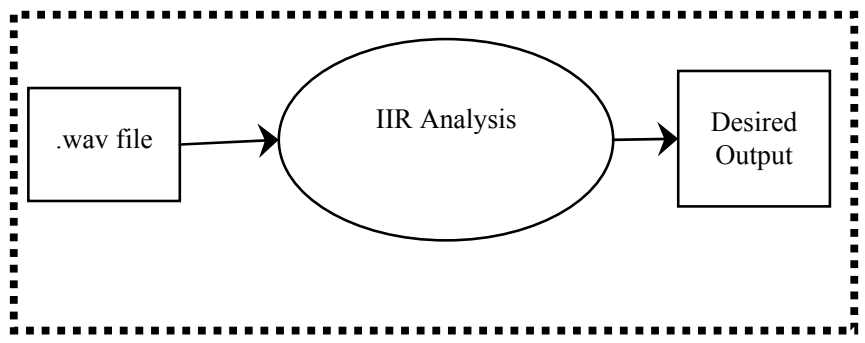

[Fig.-7: 0 Level DFD for IIR Analysis

I. Flow Chart for High Pitch Analysis 


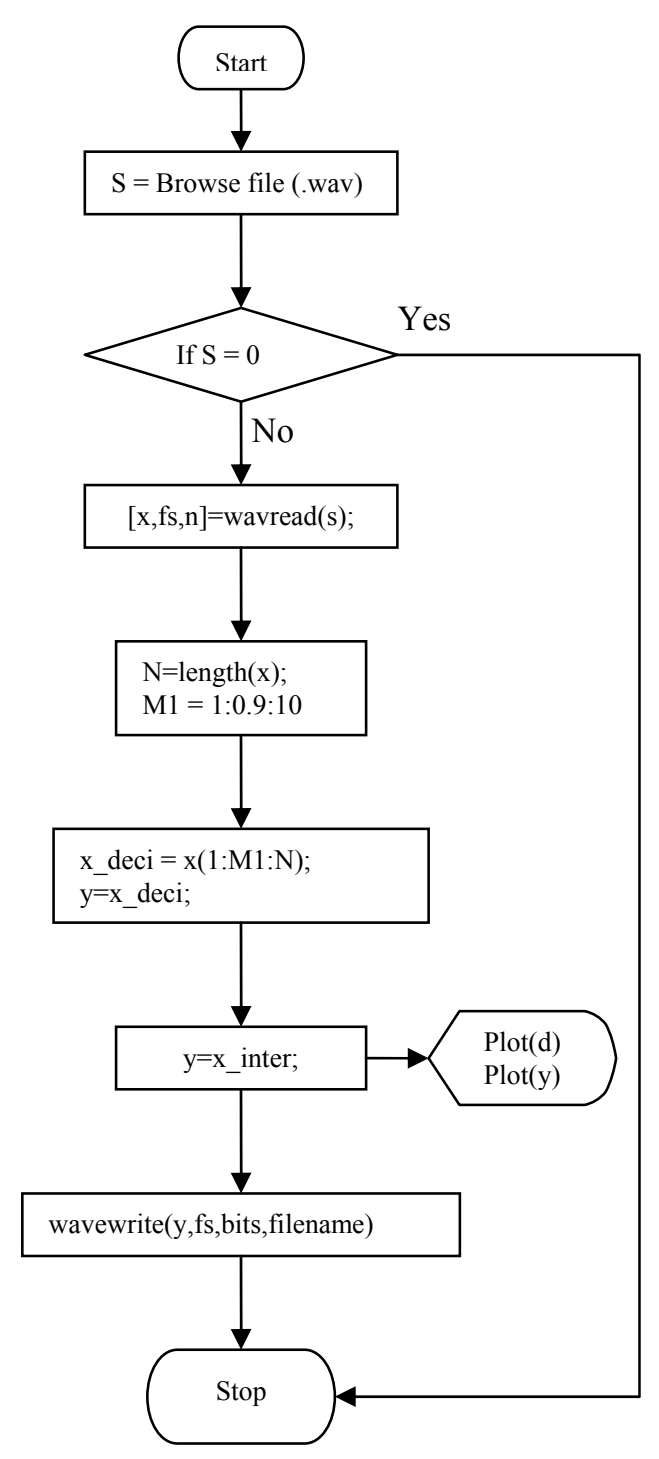

Fig-8: Flow Chart for High Pitch Analysis

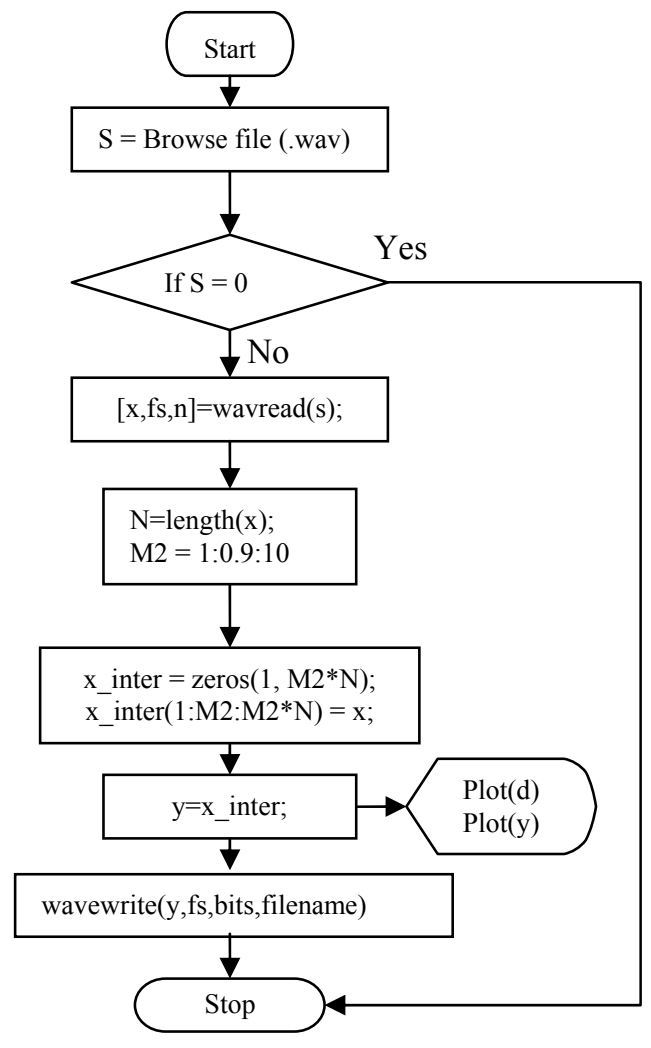

Fig-9: Flow Chart for Low Pitch Analysis

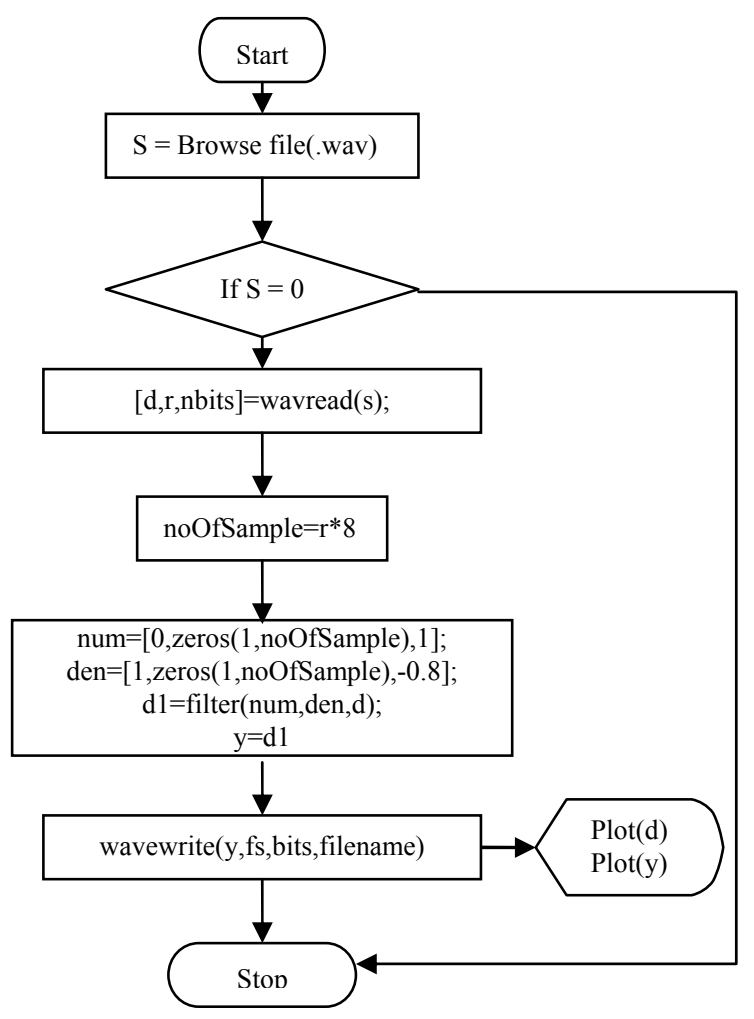

Fig-10: Flow Chart for FIIR Analysis

\section{J. Flow Chart for Low Pitch Analysis}

K. Flow Chart for FIIR Analysis

L. Flow Chart for IIR Analysis 


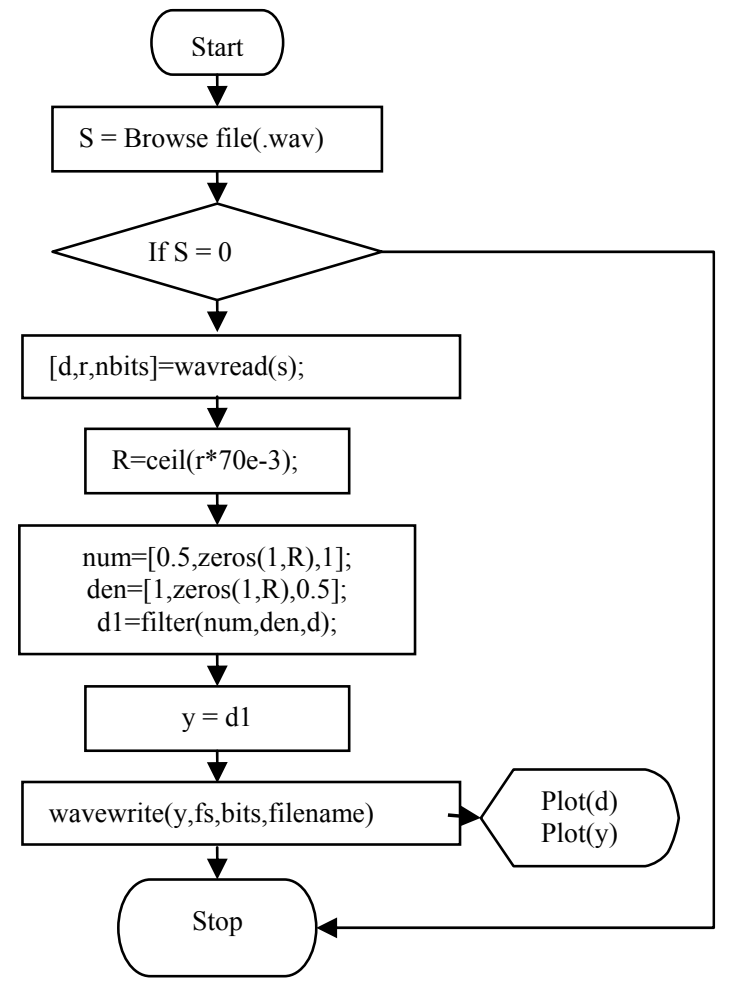

Fig-11: Flow Chart for IIR Analysis

\section{RESUlts AND ANALYSIS}

\section{A. Result Analysis for High Pitch Effect Analysis}

Based on above flow chart [Fig-8] when a compressed voice signal having an High Pitch Effect. The difference between original and effected can easily seen. Here we introduce High Pitch Effect of 0.9 sec delay, which if we want, it can be changed. The comparative Spectrum of with and without High Pitch Effect is shown in Fig. 12. The difference between without High Pitch Effect and with High Pitch Effect is clearly visible in the Fig. 12.
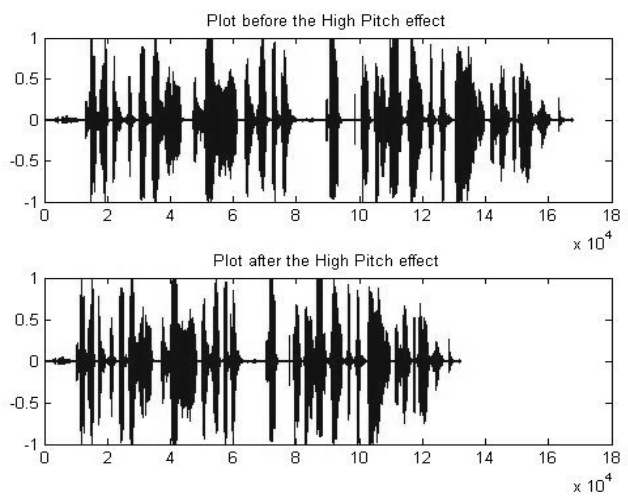

[Fig.-12: Spectrum Analysis for High Pitch Analysis]

\section{B. Result Analysis for Low Pitch Effect Analysis}

Based on above flow chart [Fig-9] when a compressed voice signal having a Low Pitch Effect. The difference between original and effected can easily seen. Here we introduce Low Pitch Effect of 0.9 sec delay, which if we want, it can be changed. The comparative Spectrum of with and without Low Pitch Effect is shown in Fig.13. The difference between without Low Pitch Effect and with Low Pitch Effect is clearly visible in the Fig. 13.
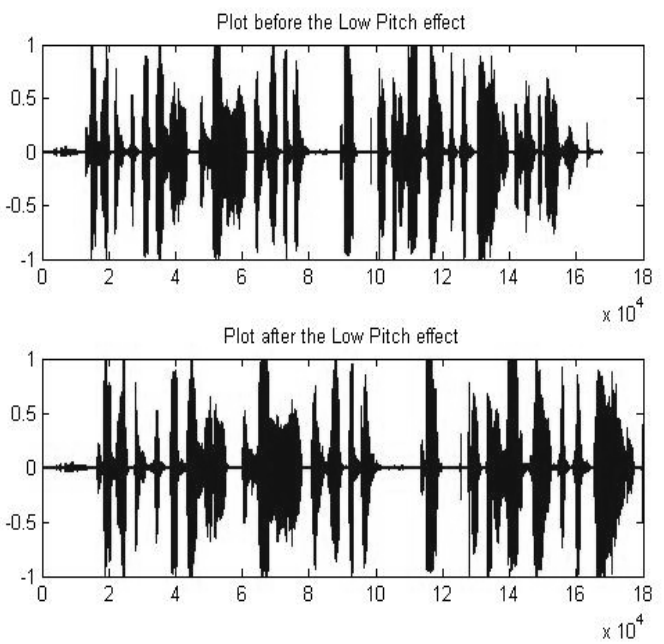

[Fig.-13: Spectrum Analysis for Low Pitch Analysis]

\section{Result Analysis for FIIR Effect Analysis}

Based on above flow chart (Fig. 10) the compressed voice signal having a FIIR effect. The difference between original and effected wave can be readily seen. Here, we have introduced the FIIR effect. The comparative Spectrum of with and without FIIR effect is shown in Fig. 14. The difference between without FIIR effect and with FIIR effect is clearly visible in the Fig. 14.
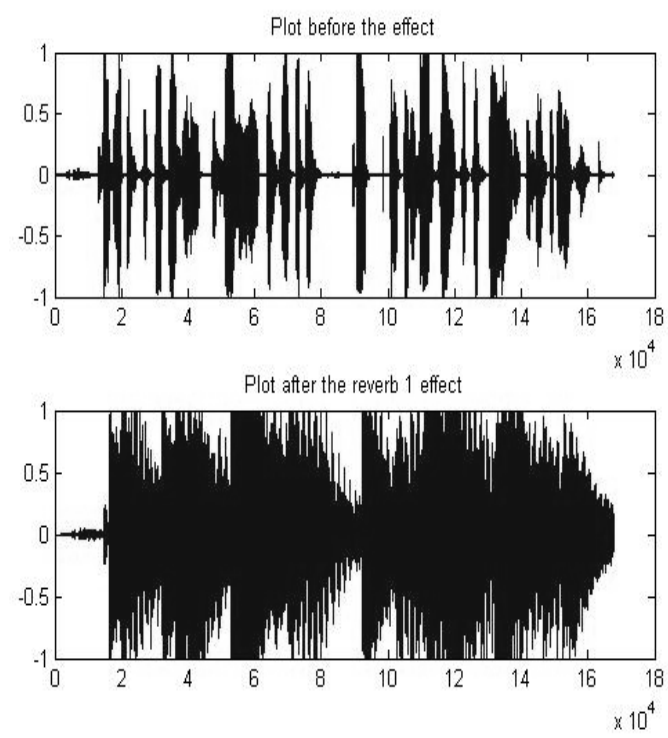

[Fig.-14: Spectrum Analysis for FIIR Effect Analysis]

\section{Result Analysis for IIR Effect Analysis}

Based on above flow chart a compressed voice signal having a IIR effect and the difference between original and effected wave is clearly evident. In doing so, we have introduce IIR effect. The comparative Spectrum of with and without IIR effect is shown in fig.-[15]. The difference between without IIR effect and with IIR effect is further illustrated in Fig. 15. 

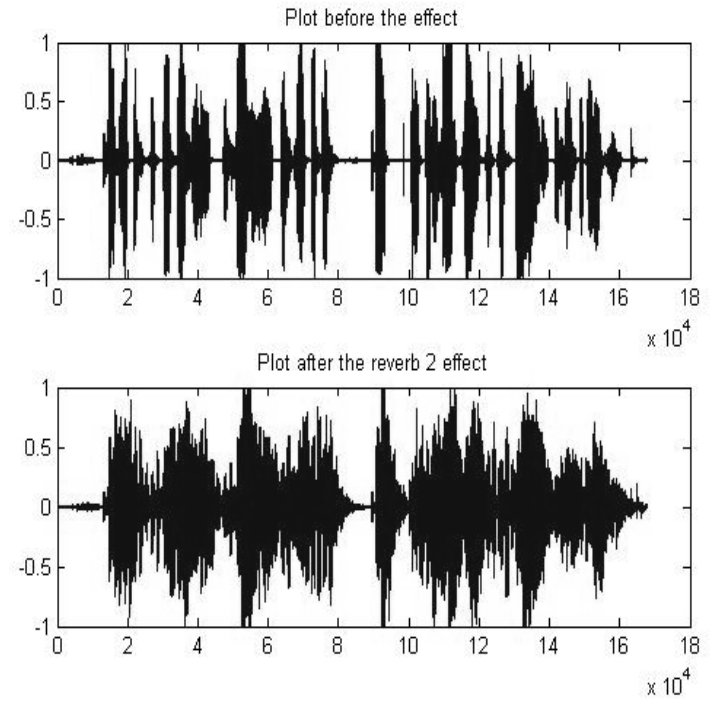

[Fig.-15: Spectrum Analysis for IIR Effect Analysis]

\section{CONCLUSION}

The present studies focus the analysis and evaluation of the High Pitch Effect, Low Pitch Effect, FIIR Effect, and IIR Effect based on spectrum of the voice signal. It is noticed that comparison based on the parameters, spectrum can clearly shows the difference between original and effected signal as illustrated in Figures [12]-[15]. We thus conclude that the variation in the VSA parameters yields a relative comparison of constitutional and unconstitutional voice of $\mathrm{N}$-bit signals can be done.

\section{REFERENCES}

[1] Vijay Kumar Chaudhari, R. K. Singh, Dinesh Varshney, Shiv Kumar, "A New Approach For Voice Spectrum Analysis (VSA): Suitable For Pervasive Computing Using Matlab", Published in IEEE International Conference ICSTE-2009-Chennai India, July-24-26, 2009, ISBN: 978-9-8142-8724-1, Page No.:- 390, Published by Word Scientific, C 2009 IACSIT

http://www.iacsit.org/proceeding/icste09.tov.pdf

[2] Shiv Kumar, Vijay Kumar Chaudhari, R. K. Singh, Dinesh Varshney, "A New Algorithm for Voice Signal Compression (VSC) and Analysis Suitable for Limited Storage Devices Using MatLab", Published in International Journal of Computer and Electrical Engineering (IJCEE) on Dec-2009, ISSN Print: 1793-8163, ISSN Online: 1793-8198, Volume-1, Number-5, Page No.: 684, Published By: IACSIT, (C) 2009 IACSIT

http://www.ijcee.org/content.htm

[3] Shaleena Jeeawoody, "Voice Analysis and Recognition as a Car Theft Deterrent", California State Science Fair 2008, Project No: J1307, Ap2/08 http://www.USC.edu/CSSF/Current/Projects/J1307.pdf

[4] Khalid Saeed, "Sound and Voice Verification and Identification a Brief Review of Töeplitz Approach", Znalosti 2008, pp. 22-27, ISBN 978-80-227-2827-0.FIIT STU Bratislava, .stav informatiky a softvÈrovĖho inæinierstva, 2008 http://znalosti2008.fiit.stuba.sk/download/articles/znalosti2008-Saeed. pdf

[5] Maria Markaki, Andre Holzapfel, Yannis Stylianou "Singing Voice Detection using Modulation Frequency Features", Computer Science Department, University of Crete, Greece http://www.sapa2008.org/papers/122.pdf

[6] Engin Avci, "An Automatic System for Turkish Word Recognition Using Discrete Wavelet Neural Network Based on Adaptive Entropy", International Conference-Elazig, Turkey-Dec 06, 2007, the Arabian Journal for Science and Engineering, Volume 32, Number 2B. http://www.kfupm.edu.sa/publications/ajse/Articles/322B_P.04.pdf
[7] Frank A Russo, Lola L Cuddy, Alexander Galembo, William Forde Thompson, "Sensitivity to tonality across the pitch range", Perception, 2007, volume 36, pages 781-790, DOI: $10.1068 / \mathrm{p} 5435$, ISSN 0301-0066

(print) http://www.perceptionweb.com/perception/fulltext/p36/p5435.pdf

[8] Murtaza Bulut, "Mult-level emotional speech analysis resynthesis", Proposed thesis for Ph.D. in Electrical Engineering, University of Southern California (USC), Los Angeles, CA, (graduation expected in November 2007). http://sail.usc.edu/ mbulut/pdfs/murtaza_bulut_CV_Oct2007.pdf

[9] Michael I Mandel, "Recognition and Organization of Speech and Audio (LabROSA)", Ph.D. candidate in the department of Electrical Engineering at Columbia University, Proposed work for 2009 (Jan) exp. http://mr-pc.org/cv.pdf

[10] Sarantos Psycharis "The Didactic Use of Digital Image Lossy Compression Methods For The Vocational Training Sector", University of Agean, Proceeding of Current Developments in Technology-Assisted Education 2006(FORMATEX 2006) http://www.formatex.org/micte2006/pdf/2065-2069.pdf

[11] Graziano Bertini, Federico Fontana, Diego Gonzalez, Lorenzo Grassi, Massimo Magrini "Voice Transformation Algorithms With Real Time Dsp Rapid Prototyping Tools", Proceeding In International Conference -Eusipco 2005 http://www.eurasip.org/Proceedings/Eusipco/Eusipco2005/defevent/p apers/cr1227.pdf

[12] Marcos Faundez-Zanuy, "Non Liner speech Processing", Proceeding in ISCpad 66, 19-22 April-2005, Barcelona, Spain http://www.isca-speech.org/escapads/pad67.text

[13] Keikichi HIROSE, "Speech Prosody 2004", Proceeding in ISCApad 56, International Conference: Speech Prosody 2004 on March 23 -26, 2004-Japan, http://www.gavo.t.u-tokyo.ac.jp/sp2004

[14] Kyogu Lee,"Pitch Perception: Place Theory, Temporal Theory, and Beyond", IEE 391 Special Report (Autumn 2004), Center for Computer Research in Music and Acoustics (CCRMA), Music Department, Stanford University. http://ccrma-www.stanford.edu/ kglee/pubs/klee_ee391_fall04.pdf

[15] Bal'azs Bank, Federico Avanzini, Gianpaolo Borin, Giovanni De Poli, Federico Fontana, Davide Rocchesso, "Physically Informed Signal Processing Methods for Piano Sound Synthesis: A Research Overview", EURASIP Journal on Applied Signal Processing 2003:10, 941-952, C2003 Hindawi Publishing Corporation. http://home.mit.bme.hu/ bank/publist/jasp03.pdf

[16] Douglas A. Reynolds, Larry P. Heck, "Automatic Speaker Recognition" Presented at the AAAS 2000 Meeting Humans, Computers and Speech Symposium 19 February 2000, Nuance Communications United States Air Force. http://www2.imm.dtu.dk/ 1f/Automatic\%20Speaker\%20Recognition. pdf

[17] Matti Karjalainen, Tero Tolonen,"Multi-Pitch and Periodicity Analysis Model for Sound Separation and Auditory Scene Analysis", Laboratory of Acoustics and Audio Signal Processing-Helsinki University of Technology http://www.ee.columbia.edu/ dpwe/papers/KarjT99-pitch.pdf

[18] Ganesh K Venayagamoorthy, Viresh Moonasar, Kumbes Sandrasegaran, "Voice Recognition Using Neural Networks",Institute of Information Science and Technology (IIST), Massey University, New Zealand, Published in IEEE in 1998, Val. No. 0-7803-5054-5.0029. http://acil.mst.edu/documents/voice_recognition_using.pdf

[19] Brett A. St. George, Ellen C. Wooten, Louiza Sellami "Speech Coding and Phoneme classification Using MATLAB and NeuralWorks" Department of Ellectrical Engineering,U.S. Naval Academy-Annapolis, MD 21402. http://file.engrng.pitt.edu/fie97/papers/1037.pdf

[20] Audio Signal Processing and Coding "Andress Sparias Ted Painter Venkatraman"

http://www.amazon.com/Signal-Processing-Coding-Andreas-Spanias/ $\mathrm{dp} / 0471791474$ 


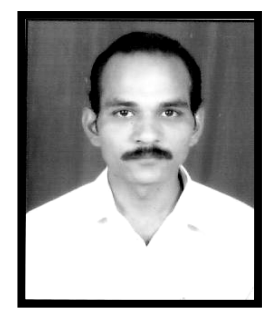

Vijay K Chaudhari received B.E. (Electronics) degree from S.S.T C.O.E.T. -Jalgaon (M.S.). Later on, he completed C.DAC. (Software Application) from SIST-Chennai (A.P.), and received M. Tech. (Electronics) from MANIT-Bhopal (M.P.). Presently he is Head and Asst. Professor in the Department of Information Technology at Technocrat Institute of Technology-Bhopal (M.P.)-India since 2001. He is pursuing Ph.D. in Computer Science form Madhya Pradesh Bhoj University-Bhopal. He has made outstanding contribution in the field of Network Security, Signal Processing, Synchronous Digital Hierarchy, Image Processing, Signal Frequency Spectrum Analysis, and Video Mining. He has around 31 papers published in different International/National Conferences and Journal, out of which 06 are International Journals, 15 papers in International Conference proceeding, 10 papers in National Conference proceeding, 3 papers are also listed in IEEE Xplore Digital Library. He has successfully supervised 63 projects for B.E. students and 5 projects for M. Tech. students. He has 9 years experience in the field of research and professional education.

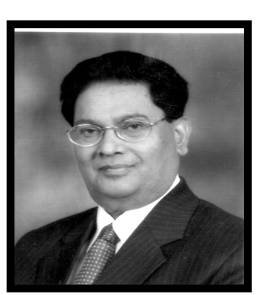

Dr. R.K. Singh received Ph.D. degree from the B.H.U.-India in 1969. Prof. Singh is the first recipient of Dr. Kailash Nath Katju Award for Sciences, presented by the President of India in 1984 for outstanding contributions in condensed matter physics. Prof. Singh has published over 650 research papers in Int. Journals/Conf. Proceedings and 2 Books (edited). He has visited several countries to deliver lectures as visiting Professor and participate \& chair conferences. He has supervised $62 \mathrm{Ph} . \mathrm{D}$. Scholars in physics \& computer science. Prof. Singh established School of Physics as its Founder Professor and Head at Barkatullah University Bhopal (M.P.)-India from 1984-95 and got sanctioned \& implemented Superconductivity, Special Assistance and Electronics Programmes from the University Grants Commission. As Hon. Director, he established a Centre for Science \& Technology Development Studies at MP Council of Science \& Technology. As Founder Director, he established the Computer Centre and CS Department. He was also Head of the University Science Instrumentation Centre at Barkatullah University, Bhopal from 1984 - 95. As the Vice Chancellor, Guru Ghasidas University-Bilaspur-India for above two terms (June 22, 1995 to Jan 22, 2002), Prof. Singh introduced 50 courses and established 8 new Institutes. Prof. Singh has served the M. P. Bhoj (Open) University, Bhopal as Vice Chancellor (Jan 23, 2002 to Jan 4, 2005) with his wide experience and vision of establishing an Institute of Distance Education at GGU. He was elected the Member of the Executive Council of the Association of Commonwealth Universities for 2002-03 and 2003-04, elected as Vice President of the Int. Academy of Physical Sciences for 2004-06. Prof. Singh is made outstanding contribution in the field of physics, computer \& computational mathematics.

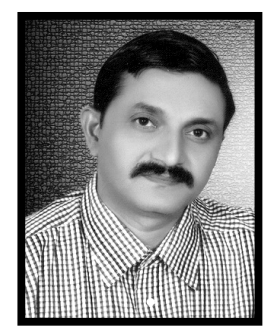

Dr. Dinesh Varshney is currently HEAD School of Instrumentation and Professor in Physics. Dr. Varshney took his M. Sc. in 1988, M. Phil in 1989 on Condensed matter Physics and Ph. D. in 1994 on Materials Science from Barkatullah University Bhopal. Credited by National ISCA Young Scientist award for Materials Science 1998, Int. Academy of Physical Sciences Young Scientist awards for Physics 1997 and M. P. State Young Scientist award for Physics 1994, Best research Scientist award of the University 2009. He is a recipient of Int. Centre for Theoretical Physics, Italy fellowship for the year 1998-99. Prof. Varshney visited ICTP, Trieste, Loughborough University, IRC Cambridge U. K. and IAFM Pisa for group discussion meetings. Prof. Varshney has a research and teaching experience of over 20 years and is author of over 125 papers in journals of Int. repute, 2 review articles and supervised 15 students for their $\mathrm{Ph}$. D. work. He is a Coordinator M. Sc. Materials Science programme sanctioned by U.G.C. under Innovative programme. Prof. Varshney is Regional Director, Multimedia regional center-Indore, M.P. Bhoj (open) University. He is actively engaged in information theory and its application as quality improvement analysis for audio, video and data transfer for Edusat establishment, Voice signal compression and spectrum analysis, Development of an efficient and intelligent data retrieval model for Semantic web, Identification of educational performance indicator as a data mining probe for higher education.

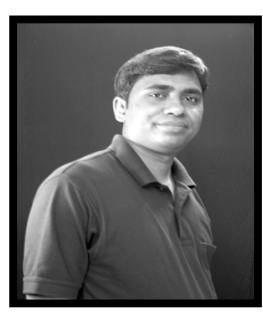

Shiv Kumar received Diploma (Leather Technology) from Govt. Lather Institute of Agra (U.P.)-India in year 2000. He worked as a Tanner in Ajaction Lather Punjab Ltd. Punjab in between 2000 to 2001. After that he completed B. Tech. (Information Technology) degree from Bhagwant Institute of Technology (U.P.)-India in year 2004. $\mathrm{He}$ has worked as a lecturer in C.S.E. / I.T. department at CEC-Bilaspur (C.G.)-India in between Dec-2004 to April-2006 and SSCET-Bhilai (C.G.)-India in between May-2006 to August-2006. Now he is pursing M. Tech. (Information Technology) from Technocrat Institute of Technology-Bhopal (M.P.) India. His research interests include Voice Signal Compression, Tonality Computation, Image Processing, and Spectrum Analysis of Signal. He is having 7 papers in International Conferences, 6 papers in National Conferences and 4 papers in IEEE-Xplore and IEEE CS digital library, 2 Papers in International Journal. He has made outstanding contribution in the field of voice signal compression and spectrum analysis. 